\title{
ARTICLE OPEN Realizing and manipulating space-time inversion symmetric topological semimetal bands with superconducting quantum circuits
}

\author{
Xinsheng Tan ${ }^{1}$, Yuxin Zhao ${ }^{2,3}$, Qiang Liu' ${ }^{1}$ Guangming Xue ${ }^{1}$, Haifeng $\mathrm{Yu}^{1,4}$, Z. D. Wang ${ }^{2}$ and Yang $\mathrm{Yu}^{1,4}$
}

Symmetries of space-inversion $(P)$, time-reversal $(T)$, as well as the joint space-time inversion $(P T)$ are fundamental and significantly important in physics. Here we have experimentally realized the joint $P T$ invariant $\mathbb{Z}_{2}$-type topological semimetal-bands, via an analogy between the momentum space and a controllable parameter space in superconducting quantum circuits. By measuring the whole energy spectrum of the system, we clearly imaged an exotic tunable gapless band structure typical of topological semimetals. Two topological quantum phase transitions, from a topological semimetal to two kinds of insulators, can be manipulated by continuously tuning the different parameters in the experimental setup, one of which captures the $\mathbb{Z}_{2}$ topology of the $P T$ semimetal via merging a pair of nontrivial $\mathbb{Z}_{2}$ Dirac points. Remarkably, the topological robustness was demonstrated unambiguously, by adding a perturbation that breaks only the individual $T$ and $P$ symmetries but keeps the joint $P T$ symmetry. In contrast, when another kind of $P T$-violating perturbation is introduced, a topologically trivial insulator gap is fully opened.

npj Quantum Materials (2017)2:60; doi:10.1038/s41535-017-0062-3

\section{INTRODUCTION}

Symmetry and topology, as the two fundamentally important concepts in physics and mathematics, have not only manifested themselves in science, but also provided us a profound understanding of arresting natural phenomena. Recently, topological gapless systems, such as Weyl semimetals ${ }^{1-3}$ and $\mathbb{Z}_{2}$ topological metals/semimetals, ${ }^{4,5}$ have significantly stimulated research interest. Analogous to that in gapped topological systems, such as topological insulators and superconductors, the discrete symmetry that is rather robust against symmetry-preserved perturbations can enrich the topological physics of gapless systems as well. As is known, the discrete time-reversal $(T)$, space-inversion $(P)$, and charge-conjugate $(C)$ symmetries are fundamental and intriguing in nature. For examples, in high energy physics, any local quantum field theory must preserve the joint CPT symmetry, which is required by the unitarity and Lorentz invariance of the theory, and the source of $C P$ violation still remains as one of seminal mysteries in the Standard Model. While in condensed matter systems, it is ubiquitous that $P, T$ and $C$ impose constraints on band structures and lead to new topological classifications of band theories. ${ }^{6-8}$ Among various combinations of $P, T$ and $C$, the joint $P T$ symmetry actually inverts the space-time coordinates $x^{\mu} \rightarrow-x^{\mu}$ with $\mu=0,1$, 2,3 and $x^{0}=t$, and therefore evidences themselves to be fundamental and significant in physics.

Recently, a theory of PT-invariant topological gapless bands has rigorously been established, ${ }^{9}$ through revealing a profound connection between the joint $P T$ symmetry and an elegant $K O$ theory of algebraic topology. ${ }^{10}$ The physical manifestation of PT symmetry in band theories can simply be seen from the commutation relation as $[\widehat{A}, H]=0$, where $H$ is the system
Hamiltonian, and the joint $P T$ symmetry is represented by an anti-unitary operator $\widehat{A}$. When $\widehat{A}^{2}=1$, the topological classification of band-crossing points in two-dimensional band structures corresponds to the reduced $K O$ group, $K O\left(S^{1}\right) \cong \mathbb{Z}_{2}$, which implies that there exist band-crossing points having nontrivial $\mathbb{Z}_{2}$ topological charges in two dimensions. ${ }^{9}$ Although the $K O$ theory of algebraic topology seems to be rather abstruse for most physicists, the predicted topological band crossing points can be realized in a simple but representative dimensionless Hamiltonian, which is explicitly given by ${ }^{9}$

$H(k)=\sin k_{x} \sigma_{2}+\left(\lambda \pm \cos k_{y}\right) \sigma_{3}$,

where $\sigma_{j}$ is the $j$ th Pauli matrix and $k_{x, y}$ denotes the twodimensional wave vector, and $\lambda$ is a tunable parameter. For the above system, the joint $P T$ symmetry can be denoted by the operator $\widehat{A}=\sigma_{3} \hat{\mathcal{K}}$ that commutates with the Hamiltonian in Eq. (1), where $\sigma_{3}$ corresponds to the $P$ operation, and $\hat{\mathcal{K}}$, defined as the complex conjugate, denotes the time-reversal $T$ operation. When $-1<\lambda<1$, the Hamiltonian in Eq. (1), which actually describes a topologically nontrivial spin(1/2)-orbital quantum system in two dimension, has four band-crossing points possessing the $P T$-protected $\mathbb{Z}_{2}\left(\nu_{\mathbb{Z}_{2}}=1\right)$ topological charges; while they are topologically trivial for $|\lambda|=1$ because a pair of $\mathbb{Z}_{2}$ points merge to one point, and the gap opens for $|\lambda|>1$ (some details will be addressed later). It is noted that although the Hamiltonian in Eq. (1) has both $\hat{P}=\sigma_{3} \hat{i}$ and $\hat{T}=\hat{\mathcal{K}} \hat{i}$ symmetries, with $\hat{i}$ being the inversion of the wave vector $k$, the topological stability of these band-crossing points merely requires the joint $P T$ symmetry according to the PT invariant topological band theory, namely, the

\footnotetext{
${ }^{1}$ National Laboratory of Solid State Microstructures, School of Physics, Nanjing University, Nanjing 210093, China; ${ }^{2}$ Department of Physics and Center of Theoretical and Computational Physics, The University of Hong Kong, Pokfulam Road, Hong Kong, China; ${ }^{3}$ Max-Planck-Institute for Solid State Research, D-70569 Stuttgart, Germany and ${ }^{4}$ Synergetic Innovation Center of Quantum Information and Quantum Physics, University of Science and Technology of China, Hefei, Anhui 230026, China Correspondence: Haifeng Yu (hfyu@nju.edu.cn) or ZD Wang (zwang@hku.hk) or Yang Yu (yuyang@nju.edu.cn)
}

Received: 5 March 2017 Revised: 18 September 2017 Accepted: 29 September 2017

Published online: 01 November 2017 
$T / P$-symmetry is allowed to be broken individually while the $P T$ topological protection still remains.

Although the experimental demonstration of a topological gapless band with PT symmetry would significantly deepen our understanding of topological quantum matter, a PT-symmetryprotected topological gapless band has yet to be observed in real materials. Artificial superconducting quantum circuits possessing high controllability ${ }^{11-15}$ can provide an ideal and powerful tool for quantum simulation and the study of novel quantum systems, ${ }^{16-20}$ including topological ones. ${ }^{21,22}$ In this paper, we have experimentally realized novel PT-symmetry-protected topological semimetal-bands that represent a gapless spectrum on a square-lattice, via an analogy between momentum space and a controllable parameter space in superconducting quantum circuits. By measuring the whole energy spectrum of our system, we have clearly imaged an exotic tunable gapless band structure typical of topological semimetals, shown as nontrivial $\mathbb{Z}_{2}$-type Dirac points in momentum space. The two new distinct quantum phase transitions from a topological semimetal to two different insulators can be manipulated by continuously tuning the different parameters in the simulated effective Hamiltonian, particularly one of which exhibits the $\mathbb{Z}_{2}$ topology in the $P T$ semimetal via merging a pair of nontrivial $\mathbb{Z}_{2}$ Dirac points. Furthermore, to demonstrate unambiguously the topological robustness of PT symmetry, a perturbation that breaks only the individual $T$ and $P$ symmetries is intentionally added, with the joint $P T$ symmetry being still preserved. It is verified by experimental data that the Dirac points of the topological semimetal-bands are still present under such perturbations, though the point positions and the band pattern are changed drastically. However, when another kind of perturbation is added to break the PT symmetry in our experiment, the energy gap is fully opened and the Dirac points disappear completely, showing the essential role of $P T$ symmetry underlying the topological robustness. All of these illustrate convincingly the topological protection of $P T$ semimetals. Notably, the present work is the first experimental realization and manipulation of fundamental space-time inversion symmetric topological semimetal-bands (without individual $T$ and $P$ symmetries) in nature, which opens a window for simulating and manipulating topological quantum matter.

\section{RESULTS}

The superconducting quantum circuits used in our experiment consist of a superconducting transmon qubit embedded in a three dimensional aluminum cavity. ${ }^{23-30}$ The transmon qubit, which is composed of a single Josephson junction and two pads $(250 \mu \mathrm{m} \times$ $500 \mu \mathrm{m})$, is patterned using standard e-beam lithography, followed by double-angle evaporation of aluminum on a $500 \mu \mathrm{m}$ thick silicon substrate. The thicknesses of the Al film are 30 and 80 $\mathrm{nm}$, respectively. The chip is diced into $3 \mathrm{~mm} \times 6.8 \mathrm{~mm}$ size to fit into the $3 \mathrm{D}$ rectangular aluminum cavity with the resonance frequency of TE101 mode $9.053 \mathrm{GHz}$. The whole sample package is cooled in a dilution refrigerator to a base temperature $30 \mathrm{mK}$. The dynamics of the transmon is well described by the theory of circuit QED, which has been developed for discussing the combined system of artificial atoms and microwave fields. ${ }^{23,26,31}$ We designed the energy level of the transmon qubit to let the system work in the dispersive regime. The quantum states of the transmon qubit can be controlled by microwaves. Inphase quadrature (IQ) mixers combined with $1 \mathrm{GHz}$ arbitrary wave generator (AWG) are used to adjust the amplitude, frequency, and phase of microwave pulses. To read out qubit states, we use ordinary microwave heterodyne setup. The output microwave is pre-amplified by HEMT at $4 \mathrm{~K}$ stage in the dilution refrigerator and further amplified by two low noise amplifiers at room temperature. The microwave is then heterodyned into $50 \mathrm{MHz}$ and collected by ADCs. The measurement is performed with so called
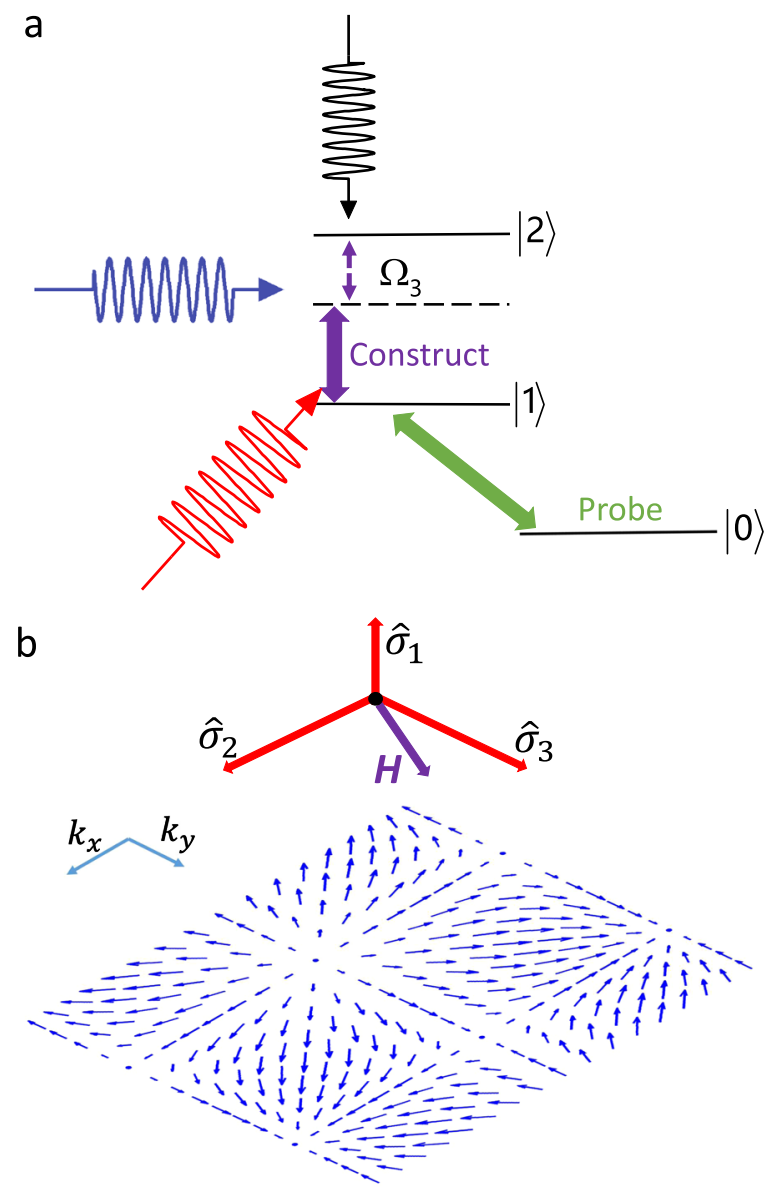

Fig. 1 Experimental scheme for the realization of the lattice Hamiltonian. a States $|2\rangle$ and $|1\rangle$ of a transmon are used as the energy levels of an artificial spin- $1 / 2$ particle, whose three components may be denoted by the three Pauli matrices $\hat{\sigma}_{1,2,3} .|0\rangle$ is chosen as an ancillary level to probe the eigenvalues of a Hamiltonian. Microwaves with various frequencies, phases, and amplitudes are applied for the construction of a semimetal Hamiltonian and circuit QED readout, respectively. The detail of the qubit manipulation can be found in the supplementary. $\mathbf{b}$ The constructed Hamiltonian is implemented by modulating the microwave amplitude, frequency, and phase, mapping to the momentum space of a square lattice

"high power readout" scheme. ${ }^{32}$ When we send in a strong microwave on-resonance with the cavity, the transmitted amplitude of the microwave will reflect the state of the transmon due to the non-linearity of the cavity QED system (please see the supplementary for the detail of experimental setup and sample calibration).

According to the circuit QED theory, the coupled transmon qubit and cavity exhibit anharmonic multiple energy levels. In our experiments, we use the lowest three energy levels, as shown in Fig. $1 a$, namely, $|0\rangle,|1\rangle$, and $|2\rangle$. The two states $|2\rangle$ and $|1\rangle$ behave as an artificial spin-1/2 particle, whose three components may be denoted by the three Pauli matrices $\sigma_{1,2,3}$ which can couple with the microwave fields. $|0\rangle$ is chosen as an ancillary level to probe the energy spectrum of the simulated system. First of all, we calibrated the transmon carefully. The transition frequencies between different energy levels are $\omega_{10} / 2 \pi=7.17155 \mathrm{GHz}, \omega_{21} /$ $2 \pi=6.8310 \mathrm{GHz}$, respectively, which are independently determined by saturation spectroscopy. The energy relaxation times of the system are $T_{1}^{01} \sim 15 \mu \mathrm{s}$ and $T_{1}^{12} \sim 12 \mu \mathrm{s}$. The dephasing times are $T_{2}^{* 01} \sim 4.3 \mu \mathrm{s}$ and $T_{2}^{* 12} \sim 3.5 \mu \mathrm{s}$. When we apply microwave drive 
along $x, y$, and $z$ directions, the effective Hamiltonian of the qubit in the rotating frame (Fig. 1b) may be written as ( $\hbar=1$ for brevity)

$\widehat{H}=\sum_{i=1}^{3} \Omega_{i} \sigma_{i} / 2$,

where $\Omega_{1}\left(\Omega_{2}\right)$ corresponds to the frequency of Rabi oscillations along $X(Y)$ axis on the Bloch sphere, which is continuously adjustable by changing the amplitude and phase of microwave applied to the system. $\Omega_{3}=\omega_{21}-\omega$, is determined by the detuning between the system energy level spacing $\omega_{21}$ and microwave frequency $\omega$. By carefully designing the waveform of AWG, we can control the frequency, amplitude, and phase of microwave.

In our experiment, we first calibrated the parameters $\Omega_{1}, \Omega_{2}$, and $\Omega_{3}$ using Rabi oscillations and Ramsey fringes. Then we design the microwave amplitude, frequency and phase to let $\Omega_{1}=0$, $\Omega_{2}\left(k_{x}\right)=\Omega \sin k_{x}, \Omega_{3}\left(k_{y}\right)=\lambda \Omega+\Omega \cos k_{y}$, with $\Omega=10 \mathrm{MHz}$ being chosen as the energy unit.

Exploiting the analogy between the above parameter space of our system and the k-space of a lattice Hamiltonian system, we now have Eq. (1) exactly. It is worth to mention that parameter $\lambda$ plays a crucial role in the realization of the PT invariant topological phase transition. To examine the band structure, we first set $\lambda=0$ and measured the entire energy spectrum of the system over the first BZ. The energy spectrum basically represents the eigenenergy as a function of $\left(k_{x}, k_{y}\right)$. Briefly, we first choose a set of $\left(k_{x}, k_{y}\right) \in$ $[-\pi, \pi) \times[-\pi, \pi)$ then apply the corresponding microwave drive to states $|1\rangle$ and $|2\rangle$. The system will form empty dressed states with eigenenergies encoding the eigenvalues of the Hamiltonian in Eq. (1). Then we turn on the probe microwave, and pump the system from $|0\rangle$ to the dressed state. We measured the resonant peak of the microwave absorption. By gradually changing $\left(k_{x}, k_{y}\right)$, we obtain the frequency of the resonant peak as a function of $k_{x}$ and $k_{y}$ (the detail of the spectroscopy measurement and data analysis can be found in the supplementary), from which we can extract the energy spectrum of the first BZ, as shown in Fig. 2. A key feature of the $P T$ invariant topological semimetal, which is the existence of nontrivial $\mathbb{Z}_{2}$-type Dirac points yielded by crossing bands, is clearly seen in Fig. 2b. These are the directly imaged Dirac cones in the experiments, indicating that we have successfully realized the topological semimetal bands that preserves the $P T$ symmetry. In addition, the positions of the Dirac points (Fig. 2a) locate at $(\pi, \pm \pi / 2)$ and $(0, \pm \pi / 2)$, agreeing well with the theoretical calculation of Eq. (1) with $\lambda=0$.

Remarkably, the present fully tunable experimental setup can also be exploited to examine the PT-protected topological stability
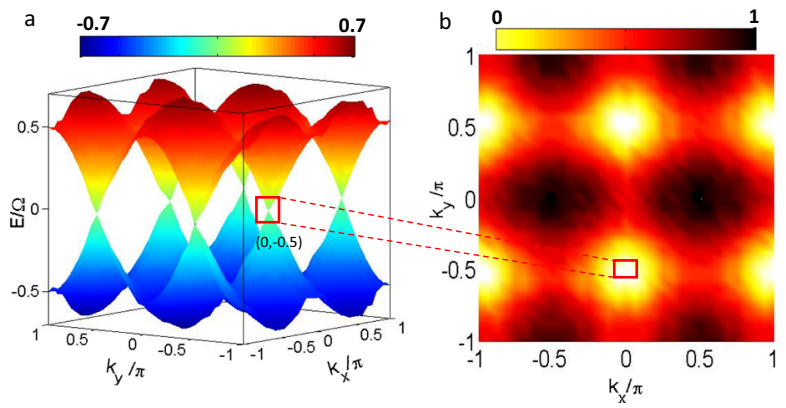

Fig. 2 Measured energy spectrum of a typical space-time inversion invariant topological semimetal. a Three-dimensional plot of the band structure of spectroscopy measurement. By tuning the driving amplitude, frequency, and phase gradually, we image the band structure of the system in the momentum space point by point. $\mathbf{b}$ Magnitude of energy gap obtained from direct measurements of the energy spectrum of the system as function of $k_{x}$ and $k_{y}$ in the first BZ. Four nontrivial $\mathbb{Z}_{2}$-type Dirac points located inside the bright regions can be observed at $(0, \pm \pi / 2),(\pi, \pm \pi / 2)$, in a full agreement with the theoretical prediction of the $\mathbb{Z}_{2}$ nontrivial band crossing points from the following aspects. First we check the topological stability of these band crossing points of nontrivial $\mathbb{Z}_{2}$ charges. From the topological band theory, each of them should be stably present under whatever perturbations that preserve the joint $P T$ symmetry and do not mix one point with another, while the individual $P$ and $T$ symmetries may be violated at the same time. ${ }^{9}$ In this experiment, we can easily realize this by introducing the perturbation $H_{1}^{\prime}=$ $\eta \sigma_{2}$ (with $\eta=1 / 2 \Omega$ being a constant) to the system. Now the parameter of $\sigma_{2}$ reads $\Omega_{2}\left(k_{x}\right)=\Omega\left(\sin k_{x}+1 / 2\right)$, which breaks both $P$ and $T$ simply because their corresponding symmetry operators $\widehat{P}=\sigma_{3} \hat{i}$ and $\widehat{T}=\hat{\mathcal{K}} \hat{i}$ do not individually commutate with the system Hamiltonian, but preserves the joint $P T$ symmetry as its symmetry operator $\widehat{A}=\sigma_{3} \hat{\mathcal{K}}$ still commutates with the Hamiltonian. We measure the spectrum for the $k_{y}=0$ plane, shown in Fig. 3a. It is observed that although the band structure is distorted dramatically, and the positions as well as neighborhood geometries of band-crossing points are changed significantly, these band-crossing points are persistently present in the first BZ without opening any gap, being perfectly consistent with the aforementioned facts of the topological band theory. On the other hand, however, when another kind of perturbation $H_{2}^{\prime}(k)=\varepsilon \sigma_{1}$ (e.g., a constant $\epsilon \sim 0.5 \Omega$ ) is introduced to the original Hamiltonian, $\hat{H}=\Omega / 2 \sigma_{1}+\Omega \sin k_{x} \sigma_{2}+\left(\lambda \Omega+\Omega \cos k_{y}\right) \sigma_{3}$, it is clear that the $P T$ symmetry is violated, since such perturbations break $P$ but preserves $T$. Accordingly the topological protection, which requires the $P T$ symmetry, is discharged. ${ }^{9}$ In agreement with the theoretical prediction, a trivial insulating gap is observed to be fully opened in the spectrum, as shown in Fig. $3 \mathrm{~b}$. In order to check that the insulating phase is trivial, one may take a limit of the term $\Omega / 2 \sigma_{1}$ to infinity, under which the system stays in the same phase since there is no gap closing occurred. However, the infinite-large limit proportional to $\sigma_{1}$ obviously corresponds to a trivial insulator.

We now turn to examine the $\mathbb{Z}_{2}$ nature of the topological charge, utilizing the fully tunable advantage of our setup. The spectrum at the $k_{x}=0$ plane clearly reflects the transition. The spectroscopic data are shown in Fig. 4a for representative values of $\lambda$ at each stage of the whole process of merging and annihilation of the $\mathbb{Z}_{2}$ band-crossing points. According to general principles of topological band theory, merging two $\nu_{\mathbb{Z}_{2}}=1$ bandcrossing points nucleates a band crossing point of trivial
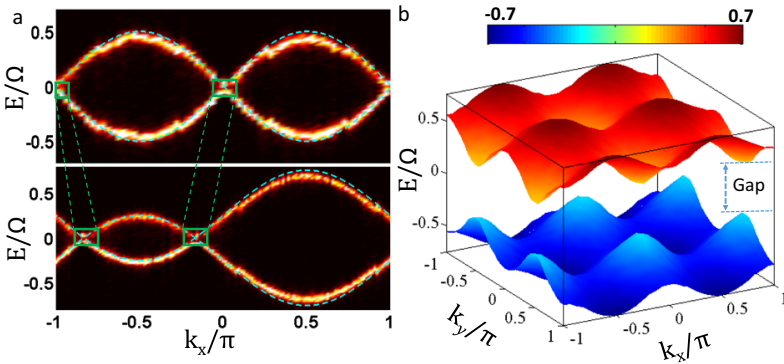

Fig. 3 Symmetry-related topological features of the Dirac points for two different but representative kinds of perturbations. a When $H_{1}^{\prime}=\eta \sigma_{2}$ is added with $\eta=0.5$ in unit of $\Omega$, which breaks both $T$ and $P$ but preserves the $P T$ symmetry, Dirac-like points still exist, though the gapless point positions are shifted (marked by the green square) and the band pattern is distorted drastically, showing the robustness of the topological nature protected by the $P T$ symmetry. Top and bottom panels correspond respectively to the cases of $\eta=0$ and $\eta=0.5$ on the plane of $k_{y}=\pi / 2$. The bright yellow and dashed green lines denote the experimental data and theoretical calculations from Eq. (1) with $H_{1}^{\prime}$ being added, respectively. b Whenever the PT symmetry is broken by adding the term $H_{2}^{\prime}=\varepsilon \sigma_{1}$ with a constant $\varepsilon(=0.5 \Omega)$, a gap is fully opened. Here $\lambda=0$ for both $\mathbf{a}$ and $\mathbf{b}$ 


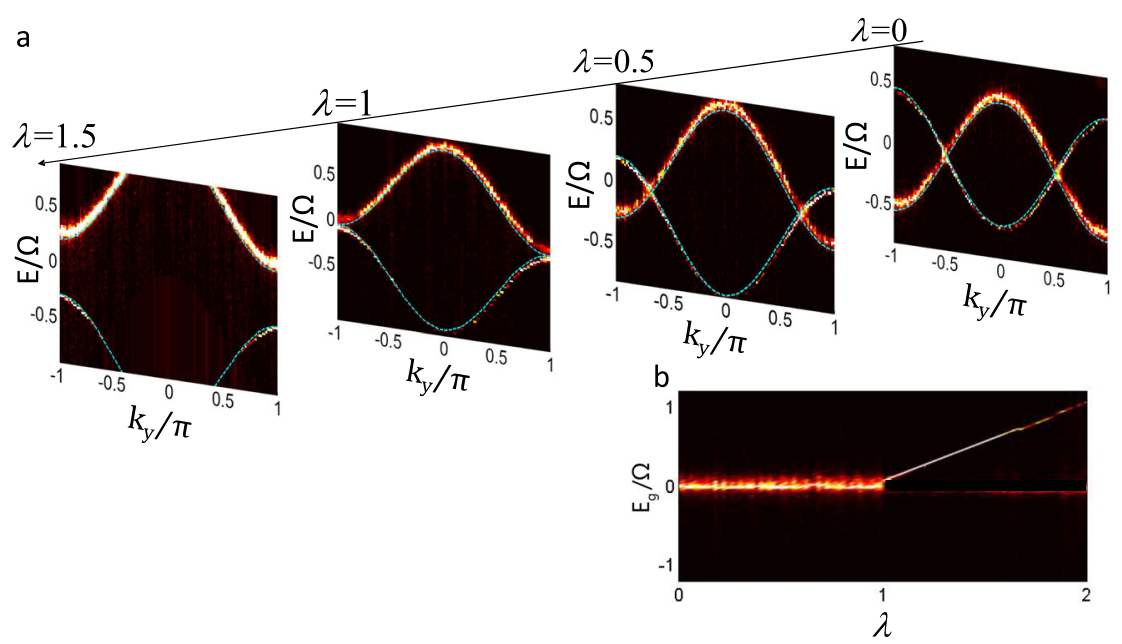

Fig. 4 Quantum phase transitions from a topological gapless semimetal to a gapped insulator as changing parameter $\lambda$. a Spectroscopy at $k_{x} \approx 0$ for various $\lambda$. From right to left $\lambda$ are $0,0.5,1$ and 1.5. It is seen that when $\lambda$ is increased from 0 to 1 , then larger than 1 , the number of Dirac-like points decreases from 4 , to 2 , then to 0 , where the gap gradually is opened, demonstrating that a topological PT invariant semimetal phase transits to a normal insulator phase. $\mathbf{b}$ Magnitude of minimum energy gap $E_{g}$ in the first Brillouin zone as a function of $\lambda$, as predicted theoretically from Eq. (1)

topological charge $\left(\nu_{\mathbb{Z}_{2}}=2 \equiv 0 \bmod 2\right)$, which can be gapped out even though the $P T$ symmetry is still preserved. ${ }^{9}$ As shown in Fig. $4 b$, we continuously increase the parameter $\lambda$ from 0 to 2 . Starting from $\lambda=0$, where two band-crossing points are well separated at $k_{y}=\pi / 2$ and $-\pi / 2$, respectively, in the onedimensional subsystem with $k_{x}=0$, the two band-crossing points are gradually moving closer and closer to each other (with regard to their distances to the $B Z$ boundaries) when $\lambda$ is increased smoothly. Then they are merged to be a new band-crossing point at the edge of the first $B Z$ for $\lambda=1$, which should be a topologically trivial point according to the topological band theory as mentioned above. Indeed, when $\lambda$ is further increased to be larger than 1, it is observed that the band crossing point of a trivial topological charge is gapped out, leading to a topologically trivial insulator that has even the PT symmetry, which verifies the aforementioned theoretical prediction. Similarly, the topological triviality follows from the same argument as that in Fig. 3b, but for the infinite limit of $\lambda \sigma_{3}$.

\section{DISCUSSION}

As shown clearly in ref. 9, the $P T$-symmetry-protected topological gapless bands belong to a new class of topological ones that are essentially different from those having the time-reversal and/or charge-conjugate symmetries, and may be even more fundamental and interesting. ${ }^{4}$ Experimental demonstration of these PT symmetry protected topological properties will significantly deepen our understanding of topological quantum matter. However, there are several big challenges that hinder the realization and investigation of the topological properties of this kind of Hamiltonian in real materials or manybody systems. The first is how to synthesize the materials with a designated Hamiltonian. Secondly, even if one is fortunate enough to have such kind of real materials, it seems extremely hard to tune the relevant parameters continuously for studying fruitful topological properties including various topological quantum phase transitions. Moreover, it seems quite difficult in experiments to directly image the whole momentum-dependent electronic energy spectrum of a bulk condensed matter system, noting that only a part of the electronic spectra (or information of Fermi surfaces/ points) may be inferred from the angle-resolved photoemission spectroscopy data (or quantum oscillation measurements). Therefore, it is important to use artificial quantum systems, like superconducting quantum circuits, to simulate $H(k)$ faithfully and to explore the topological properties of the system. In our experiments, we have realized the Hamiltonian in Eq. (1) in a parameter (analogous to the momentum) space via implementing a fully-controllable quantum superconducting circuit, such that the band structure can be directly measured over the whole first Brillouin zone (BZ) of the square lattice, enabling us to demonstrate the unique topological nature of the corresponding semimetal-bands and to visualize some crucial properties. Our experiments demonstrate the realization and manipulation of fundamental space-time inversion invariant topological semimetal bands possessing neither $T$ nor $P$ symmetry. The non-trivial bulk topological band structures of $P T$ symmetry have directly been imaged with superconducting quantum circuits. Moreover, two exotic topological quantum phase transitions have been observed for the first time. The present work is expected to stimulate experimental and theoretical interest on various $P T$ symmetric topological metals/semimetals, paving the way for quantum-simulating novel topological quantum materials.

\section{METHODS}

The key results of our paper involve simulating the Hamiltonian and imaging the energy bands of PT-symmetry-protected topological semimetal system. Generally, metals are many-particle systems. However, physicists would like to ignore irrelevant issues and derive a simplified model Hamiltonian to describe the system. The lattice structure can be pre-diagonalized in momentum space and the dispersion $E(k)$ can be obtained. Then starting from the model Hamiltonian and dispersion, researchers have explored many properties, including topological ones. Therefore, if we can simulate the Hamiltonian and obtain its dispersion, we capture the essential physics of the system. For a realistic topological semimetal system, described by the Hamiltonian in Eq. (1), the momenta $k_{x}$ and $k_{y}$ commute with each other, and in particular commute with the system Hamiltonian. Therefore, $k_{x}$ and $k_{y}$ behave purely as the numbers, rather than the usual momentum operators in a general kind of quantum systems. In this sense, we are able to experimentally simulate $k_{x}$ and $k_{y}$ for this kind of system using two independent controllable parameters, just treating them as numbers in as that solving the Hamiltonian theoretically. Notably, when these numbers combine with spin-like operators as in the Hamiltonian in Eq. (1), their corresponding products are no longer numbers, exhibiting quantum characteristics. This is a key concept in the present work. Although we here simulated merely the simplest Hamiltonian in Eq. (1) of PT invariant topological semimetal bands, the concept itself is generally valid for other quantum systems including ones with complicated interacting and can be applied to many other 
many-body systems. In our experiments, the two precisely controllable parameters are not real momenta, but they can truthfully simulate the considered quantum systems without the loss of any real physics. Using a superconducting qubit subjected to an effective magnetic field, we obtained the exact form of the Hamiltonian in Eq. (1). Then the remaining task is to measure the dispersion of the system, which is actually the eigenenergies of the Hamiltonian as a function of $k_{x}$ and $k_{y}$. These can be determined from the spectroscopy. The spectroscopic measurement of an artificial spin-1/2 particle driven by various microwave fields is similar to conventional spectroscopy experiments. The artificial spin-1/2 particle in our experiments is a 3D superconducting transmon. The transmon coupled with cavity usually exhibits anharmonic multiple energy levels. We use the lowest three energy levels $|0\rangle,|1\rangle$ and $|2\rangle$ to do the spectroscopy. $|1\rangle$ and $|2\rangle$ are chosen to form the artificial spin-1/2 particle. Then we drive it with continuous microwaves (in practice we use a long microwave pulse instead) to simulate the Hamiltonian in Eq. (1). The interaction between qubit and applied microwave transforms $|1\rangle$ and $|2\rangle$ into microwave dressed states which are stationary empty states. Moreover, the eigenenergy of the Hamiltonian in Eq. (1) is encoded in the dressed states. Then we turn on the probe microwave to pump the system to the empty dressed states. From the frequency of the resonant peaks we can extract the eigenenergies of the driven spin-1/2 particle. The detail of the measurement setup, system modeling and procedure of extracting spectra of the Hamiltonian are given in the Supplementary Materials. Note that there is difference between our spectroscopy and the conventional spectroscopy of superconducting qubits. For conventional spectroscopy, we only use one continuous microwave to pump the system to the excited states and no probe microwave is applied. When the microwave frequency resonates with the eigenstates, the excited state will be populated as long as the microwave is turned on. Therefore, the resonant peaks reflect the bare eigenenergies of the system.

\section{Data availability}

The data that support the findings of this study are available from the corresponding author upon reasonable request.

\section{ACKNOWLEDGEMENTS}

This work was partly supported by the the NKRDP of China (Grant No. 2016YFA0301802), NSFC (Grant Nos. 91321310, 11274156, 11504165, 11474152, and 61521001), and the GRF of Hong Kong (Grant Nos. HKU173055/15P and HKU173309/16P).

\section{AUTHOR CONTRIBUTIONS}

Z.D.W., Y.Y., H.Y., and X.T. designed the experiment. The data were measured by X.T., and analyzed by X.T., Y.Z., H.Y., Y.Y., and Z.D.W. The theoretical framework was developed by Y.Z. and Z.D.W. Q.L, and G.X. fabricated the sample. X.T., Y.Z., Y.Y., H.Y., and Z.D.W. wrote the paper. Y.Y. and Z.D.W. supervised the project.

\section{ADDITIONAL INFORMATION}

Supplementary information accompanies the paper on the npj Quantum Materials website (https://doi.org/10.1038/s41535-017-0062-3).

Competing interests: The authors declare that they have no competing financial interests.

Publisher's note: Springer Nature remains neutral with regard to jurisdictional claims in published maps and institutional affiliations.

\section{REFERENCES}

1. Xu, S.-Y. et al. Discovery of a Weyl fermion semimetal and topological Fermi arcs. Science 349, 613-617 (2015).

2. Lv, B. Q. et al. Experimental discovery of Weyl semimetal TaAs. Phys. Rev. X. 5, 031013 (2015).

3. Wan, X., Turner, A. M., Vishwanath, A. \& Savrasov, S. Y. Topological semimetal and Fermi-arc surface states in the electronic structure of pyrochlore iridates. Phys. Rev. B. 83, 205101 (2011).

4. Zhao, Y. X. \& Wang, Z. D. Topological classification and stability of Fermi surfaces. Phys. Rev. Lett. 110, 240404 (2013).
5. Zhao, Y. X. \& Wang, Z. D. Novel $Z_{2}$ topological metals and semimetals. Phys. Rev. Lett. 116, 016401 (2016).

6. Volovik, G. E. Universe in a Helium Droplet. (Oxford University Press, Oxford, 2003).

7. Hasan, M. Z. \& Kane, C. L. Colloquium: topological insulators. Rev. Mod. Phys. 82, 3045-3067 (2010).

8. Qi, X.-L. \& Zhang, S.-C. Topological insulators and superconductors. Rev. Mod. Phys. 83, 1057-1110 (2011).

9. Zhao, Y. X., Schnyder, A. P. \& Wang, Z. D. Unified theory of $P T$ and $C P$ invariant topological metals and nodal superconductors. Phys. Rev. Lett. 116, 156402 (2016).

10. Atiyah, M. F. K-theory and reality. Q. J. Math. 17, 367-386 (1966).

11. Xiang, Z., Ashhab, S., You, J. \& Nori, F. Hybrid quantum circuits: superconducting circuits interacting with other quantum systems. Rev. Mod. Phys. 85, 623-653 (2012)

12. Vion, D. et al. Manipulating the quantum state of an electrical circuit. Science 296, 886-889 (2002).

13. Nakamura, Y., Pashkin, Y. A. \& Tsai, J. Coherent control of macroscopic quantum states in a single-Cooper-pair box. Nature 398, 786-788 (1999).

14. Gritsev, V. \& Polkovnikov, A. Dynamical quantum Hall effect in the parameter space. Proc. Nat. Acad. Sci. 109, 6457-6462 (2012).

15. Yu, Y., Han, S., Chu, X., Chu, S.-I. \& Wang, Z. Coherent temporal oscillations of macroscopic quantum states in a Josephson junction. Science 296, 889-892 (2002).

16. Georgescu, I. M., Ashhab, S. \& Nori, F. Quantum simulation. Rev. Mod. Phys. 86, 153-185 (2014).

17. Buluta, I. \& Nori, F. Quantum simulators. Science 326, 108-111 (2009).

18. Deng, C., Orgiazzi, J.-L., Shen, F., Ashhab, S. \& Lupascu, A. Observation of floquet states in a strongly driven artificial atom. Phys. Rev. Lett. 115, 133601 (2015).

19. You, J. Q. \& Nori, F. Atomic physics and quantum optics using superconducting circuits. Nature 474, 589-597 (2011).

20. Tan, X. et al. Demonstration of geometric Landau-Zener interferometry in a superconducting qubit. Phys. Rev. Lett. 112, 027001 (2014).

21. Schroer, M. D. et al. Measuring a topological transition in an artificial spin-1/2 system. Phys. Rev. Lett. 113, 050402 (2014).

22. Roushan, P. et al. Observation of topological transitions in interacting quantum circuits. Nature 515, 241-244 (2014).

23. Wallraff, A. et al. Strong coupling of a single photon to a superconducting qubit using circuit quantum electrodynamics. Nature 431, 162-167 (2004).

24. Koch, J. et al. Charge-insensitive qubit design derived from the Cooper pair box. Phys. Rev. A. 76, 042319 (2007).

25. You, J. Q., Hu, X., Ashhab, S. \& Nori, F. Low-decoherence flux qubit. Phys. Rev. B. 75, 140515 (2007).

26. Blais, A., Huang, R.-S., Wallraff, A., Girvin, S. M. \& Schoelkopf, R. J. Cavity quantum electrodynamics for superconducting electrical circuits: an architecture for quantum computation. Phys. Rev. A. 69, 062320 (2004)

27. Devoret, M. H. \& Schoelkopf, R. J. Superconducting circuits for quantum information: an outlook. Science 339, 1169-1174 (2013).

28. Buluta, I. M., Ashhab, S. \& Nori, F. Natural and artificial atoms for quantum computation. Rep. Prog. Phys. 74, 104401 (2011).

29. Shankar, S. et al. Autonomously stabilized entanglement between two superconducting quantum bits. Nature 504, 419-422 (2013).

30. Wang, $C$. et al. Measurement and control of quasiparticle dynamics in a superconducting qubit. Nat. Commun. 5, 5836 (2014).

31. You, J. Q. \& Nori, F. Quantum information processing with superconducting qubits in a microwave field. Phys. Rev. B. 68, 064509 (2003).

32. Reed, M. D. et al. High-fidelity readout in circuit quantum electrodynamics using the Jaynes-Cummings nonlinearity. Phys. Rev. Lett. 105, 173601 (2010). Attribution 4.0 International License, which permits use, sharing, adaptation, distribution and reproduction in any medium or format, as long as you give appropriate credit to the original author(s) and the source, provide a link to the Creative Commons license, and indicate if changes were made. The images or other third party material in this article are included in the article's Creative Commons license, unless indicated otherwise in a credit line to the material. If material is not included in the article's Creative Commons license and your intended use is not permitted by statutory regulation or exceeds the permitted use, you will need to obtain permission directly from the copyright holder. To view a copy of this license, visit http://creativecommons. org/licenses/by/4.0/.

(c) The Author(s) 2017 